\title{
Modeling of Router-based Request Redirection for Content Distribution Network
}

\author{
Erwin Harahap, Janaka Wijekoon, Rajitha Tennekoon, Fumito Yamaguchi, \\ Shinichi Ishida, Hiroaki Nishi \\ Hiroaki Nishi Laboratory, Center of Computer Science, Keio University, Japan
}

\begin{abstract}
The increase of digital data between content's servers and clients in a network causes congestion problems when downloading big web contents including files, streaming media, etc. The problem becomes pronounced when a narrow bandwidth or unexpected termination of streaming services is appeared. Content Delivery Network (CDN) is introduced to overcome such problems by redirect client's request to the best server which proximal and least loaded. However, with intensity increase of people accessing a particular content, the performance of CDN is reduced and sometimes congested. We propose an architecture to solve such problems with a new method to redirect client's request through a special router called Service-oriented Router (SoR). In this paper, several analytical studies and experiments have been conducted and the result show that router-based redirection is more effective than DNS-based redirection that is currently being used. SoR has been introduces as a content-based router and has capability to do deep packet inspection (DPI) into the packet streams and analyze them. We use SoR in our proposed method as a CDN's core-router to redirect client's request to the best server. Our experiment conducted with model and numerical analytic supported by optimal redirection probability based on delay between router and server. The experiment result confirmed that the router-based redirection reduced the response time by $23.3 \%$ compared to the DNSbased redirection.
\end{abstract}

\section{General Terms}

Applications, Network Management, Modeling, Routing, Content Distribution Networks, Queuing Networks.

\section{Keywords}

modeling, content delivery networks, service-oriented router, queuing networks, content distributions network, request routing, request redirection.

\section{Introduction}

More efficient content delivery over the web has become an important element of improving web performance. Content Distribution network (CDNs) have been proposed to maximize bandwidth, improve accessibility, and maintain correctness through content replication. With CDNs, content is distributed to cache servers located close to users, resulting in fast, reliable applications and web services for the users [1]. In order to guarantee the performance of browsing experience to user even during such overload condition, web content providers are increasingly off-loading the task of content placement and distribution to CDN such as Akamai [2], Limelight Networks [3], or Mirror Image [4]. With hundreds to thousands of servers, a large CDN has capability to provide identical content to many users efficiently and reliably. When a specific web page, video, file, or program is requested by a user, the best server is dynamically determined for eliminating the load of the main server. This distribution method optimizes the throughput of content's delivery to the users.

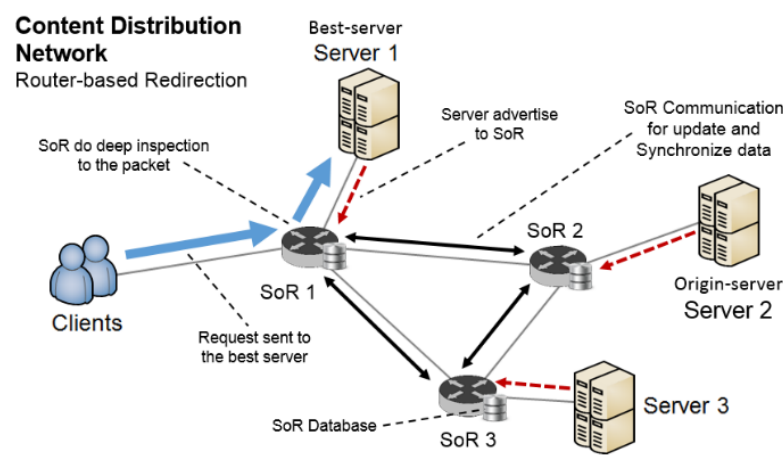

Fig 1: A proposed method. CDN with router-based redirection where regular router is replaced by Serviceoriented Router (SoR)

The definition of best server varies depending on the type of content. In general, there are two types of content in internet, namely static content or dynamic content [5]. Static content, such as images, videos, text, or pdf files are most accessible content. For this type of content, the best server is the one closest to the user. The definition of closest server is could be one which is the minimum number of hops. Network hops could be defined as the number of routers present in the path between client and server. The closest server could also be defined as the server which has the maximum network bandwidth. Alternatively, closest server could also be defined as the server with minimum network latency, or the server with lower response time and minimum server loads.

The key objective of CDN is to increase the availability of the hosted sites and to reduce the end-to-end response time between clients and servers. On reducing response time, CDNs take two approaches: (i) content placement, which involves placing the content closer to clients; (ii) request redirection, which involves forwarding client's request to the best server, including selection of the best server that can serve the requests $[6,7]$. In this paper, the method of request redirection is described, including the selection of the best server defined by minimum network delay or minimum load request processing server or combination of both parameters.

Request redirection method can be classified as either clientside or server-side redirection on the basis of the point in a request's execution where the redirection decision is made [5] A client-side redirection is one method where the client's request is redirected to a server by a client-side proxy or edge server as used in Akamai [8]. Client-side redirection is often called as DNS-based request redirection [9]. On the other hand, a server-side redirection [10] is a method where the request could be first sent to an initial destination server via existing DNS-based redirection, then the redirector in initial destination cluster redirects the request to the best server, which could be 
in either the local or the other cluster. Since it still uses a DNS resolving address in the initial process, the Server-side approach is still adopt the DNS-based redirection method.

In this paper, a new request redirection method is proposed where the request is redirected to a best server by Serviceoriented Router (SoR), which act as both regular router and redirector. SoR is a router that has capability to interact with services, and provide it to clients by using a defined application program interface (API) $[11,12,13]$. SoR can observe traffic data streams, do deep packet inspection (DPI) to the packet payload as well as packet header, and store the designated data in its database. We proposed a router-based request redirection where the mechanism is described as depicted in figure 1 . When a cluster client send request for a particular content or destination, SoR\#1 as edge-SoR or the nearest SoR to client do DPI to the packet. Next, SoR redirect the request to the best server according to its database. The data stored in database are collected and synchronized through a communication protocol between SoRs. The key objective of router-based redirection is to minimize end-to-end client delay by determining the best surrogate server whether the total latency is minimized by redirecting request to another server or served in the initial server. In particular, client requests could be first routed to initial best server as listed in SoR database. Upon arrival at the initial server, related SoRs uses a minimization response-time algorithm to determine whether to redirect to request to another server or initial server. Therefore, compared to DNS-based redirection, in SoR there is no DNS resolving address mechanism and hence reduce the total delay.

In this paper, a simple analytical model is developed to characterize the effect of router-based redirection on end-toend total delay. M/M/1 queuing networks model is used refer to Jackson networks [14] with related arrival and service rate. This analytical model allow us to perform a systematic performance evaluation of the benefits afforded by the perrequest router-based redirection method. An example finding by this model is that for any client requests, since every SoR communicate each other and updated each database, edge-SoR is aware for the request and directly forward the request to the best server, based on its database list. This shows that a routerbased redirection method can achieve better performance than DNS-based redirection which not obtain a long delay resolving server address.

The reminder of this paper is organized as follows. Section 2 provides a motivation and related work about modeling and request redirection in $\mathrm{CDN}$, while section 3 describes the Service-oriented Router. Further in section 4 a system architecture of clusters for router-based redirection is explained, and develop an M/M/1 queuing networks model refer to Jackson networks to study the architecture and section 5 present numerical studies of the redirection probability of requests forwarded to the best server and the expected response time under varying system scenarios. Section 7 provides a discussion. Finally, section 8 concludes the research with prospective future works.

\section{MOTIVATION \& RELATED WORK}

\subsection{Minimizing response time in $\mathrm{CDN}$ via DNS-based Request Redirection}

CDNs are the network system which delivers contents to users from the edges of the network, and designed to improve two performance metric: response time and system throughput [7]. Response time usually reported as a cumulative distribution of latencies which important for clients, and represent as the CDN's performance parameter. System throughput is the average number of request that can be satisfied per second. As depicted in Figure 2, refer to DNS-based redirection method,
CDNs reduces the response time by obtaining content from the nearest server (path 3) where the server address is resolved from DNS-server (path 2) compared to obtaining content from the origin (path 1).

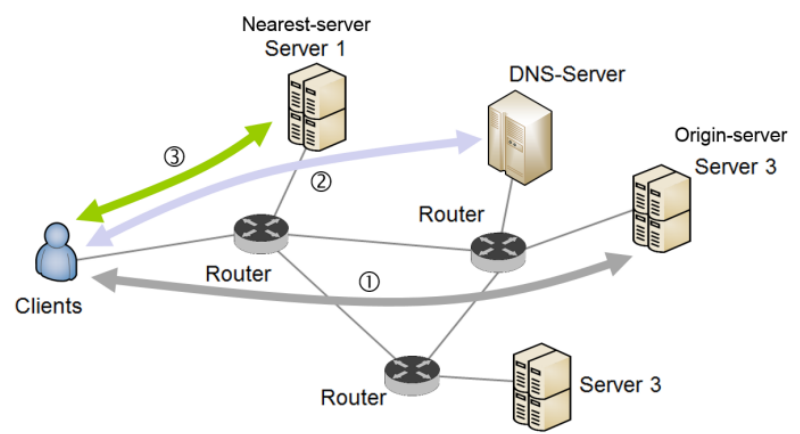

Fig 2: A General CDN Scenario. Content is replicated to the edge server by DNS-based request redirection

DNS (domain name system) is a distributed database containing records of name-to-address mapping system spread across a semi-static hierarchy of servers. Each record has a time-to-live (TTL) value that controls how long it will be cached [15]. In CDN infrastructure, DNS (dns-based redirection) is used to find the best server for a particular client's request. When the DNS-based redirection is used for the best server selection, the decision is based on the nameserver's identity, not the client's. Thus when clients and nameservers are not proximal, the DNS-based redirection method may lead to poor decisions. In addition for using DNSbased to select the best server, it requires name resolution caching to be disabled by setting TTL values to zero or very small. Small TTL values allow fine-grained load balancing and rapid response to the changes in the server or network load, but it makes clients to contact the authoritative name server for every name resolution request. This phenomenon increases web access latency and may cause congestion.

Furthermore, in DNS-based redirection method, the clients should obtain the IP address beforehand when contacting servers, then DNS traffic consumes some degrees of the bandwidth from the internet backbone, thus the time taken for connection initiation is comparatively high. This condition require a long response time when accessing the content. Therefore, minimizing the amount of DNS traffic in a network will result in effective traffic of the network

Figure 3 illustrates how a client typically finds the address of the best server using DNS-based redirection method [9]. The client application uses a resolver to make a recursive query to its local nameserver. The local nameserver may be configured statically in a system file, or dynamically using protocols like DHCP or PPP. After making the request, the client waits as the local nameserver iteratively tries to resolve the name www.example.com. The local nameserver first sends an iterative query to the root to resolve the name (steps 1 and 2), but since the subdomain example.com has been delegated, the root server responds with the address of the authoritative nameserver for the sub-domain, i.e., ns.example.com (step 3). The client's nameserver then queries ns.example.com and receives the IP address of www.example.com (steps 4 and 5). Finally the nameserver returns the address to the client (step 6 ) and the client is able to connect to the server (step 7).

Based on figure 3, in DNS-based redirection method, it needs 7 steps to find the address of the best server. This shows that the router-redirection (figure 1) has more effective method, where the address of the best server is already listed before the client send requests. Moreover, in DNS-based redirection 
method, users should get the IP address beforehand when contacting servers, then DNS traffic consumes the bandwidth of the internet backbone at some degrees, thus the time taken to connection initiation is comparatively high. This condition require a long response time when accessing the content. Our study about the DNS traffic in SINET [16], the largest Internet backbone for academics and public organizations in Japan, showed that $13.6 \%$ of the traffic is occupied by DNS in average. Therefore, minimizing the amount of DNS traffic in a network will result in effective utilization of the network. Moreover, DNS-based redirection has long response time when accessing contents. These disadvantages make considerable limitations for high-speed data communication.

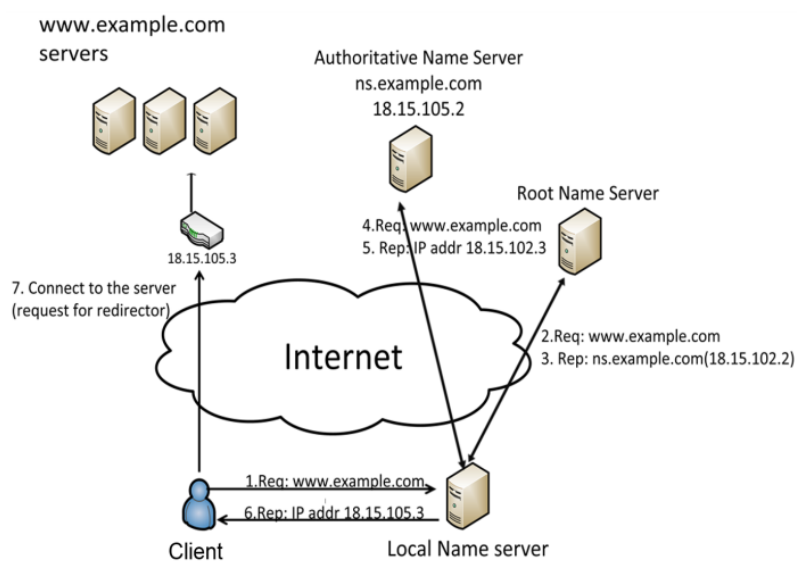

Fig 3: DNS basic operation on resolving address www.example.com

Additionally, to reduce the response time for a particular client, there are three factors to be considered: (i) network delay, (ii) server processing time, and (iii) traffic density. Our research is aimed at minimizing the above three factors by proposing a router-based request redirection as a new method of request redirection.

\subsection{Related work}

Previous research has investigated the use and effectiveness of CDNs. Recent studies confirm that CDNs reduce average download response time, but that DNS redirection techniques add noticeable overhead because of DNS latencies. Most of these studies have been empirical ones, studying and evaluating response times on real CDNs [17] [18], effectiveness of DNS redirection [17] [18] [19], server selection [20] [18], or server location [21]. Other papers and contributions have tried to model the behavior of CDNs using different techniques, but usually simplifying the nature of such systems and losing generality. These studies have focused mainly in two topics: server placement [22] or evaluation of response time. Different techniques have been used for the evaluation of the second parameter: linear time response of web servers [23], water filling schema [24] and queuing theory [25].

In this paper, the research works start from basic assumptions in queuing theory with $\mathrm{M} / \mathrm{M} / 1$ queuing model, and introduces new features of router-based redirection in $\mathrm{CDN}$ that resemble a more realistic and general model. This way the impact on performance of important parameters such as the routing redirection probability, network delay, and response time can be studied. Moreover, it is worthwhile establishing some dependencies and relationships among these parameters, as empirical analytic results are always finite and cover only a limited variability range of the $\mathrm{CDN}$ parameters.

\section{SERVICE-ORIENTED ROUTER (SoR)}

\subsection{SoR Background}

Routers are the computing systems having a great processing power, and currently take a responsibility for packet transmission and converting protocol. Although routers have been central to the IP-based communication network, for a long time routers have not been involved aggressively in the application layer where users enjoy services. Tremendous amount of packet data acquired and transmitted by routers is unique and can be considered to make web services richer (with data based on actual traffic and with wider and deeper data coverage), while data provided by service provider is currently collected by just an end host.

Service-oriented Router (SoR) is proposed and introduced as a content-based semantic router [11], a new router architecture that enables router to interact with services. It has capability to observe the traffic data stream, inspect the packet payload as well as packet headers, and stores the designated data in the associated database. SoR use a new query language SSRQL (semantic switch router query language) to operate massive traffic data stream so that application programmers can access to the database to develop a new services in a data-oriented Web2.0 world where data includes text, image, video, information of geographic location and commercial products is generated by both service providers and users. SSRQL is an extension of SQL and XML language which defines the SoR specific functionalities beside standard SQL statements.

SoR is designed to become a service-friendly router which provide unique data based on actual traffic data stream to enrich services. Router is no longer just a routing hardware that transmits data and coverts protocols, but becomes a semantic router that inspect traffic data stream including packet payloads and provides functionalities in the application later to servers, clients, and neighboring routers

The high-level design and concept of SoR are described as follows: i) while conventional routers are dealing with IP protocols, SoR deals with content in the packet payload. With richer information to determine routing table, SoR improves the efficiency and controllability of internet traffic. SoR is involved in application of P2P and overlay network by providing topology (related to information and application) in order to support fast and efficient routing; ii) seamless integration of IP-based routing and content based routing enables application programmers to create new services; iii) SoR organize a large scale distributed database among neighbor routers to improve quality of search result.

The basic form of high-level design of SoR described in two aspects: 1) massive data processing, SoR having capability of deep packet inspection (DPI) including packet header and payload, therefore the database associated with the SoR need massive data processing capability; 2) privacy issue, since database of SoR is accessible by application programmers and service providers, privacy protection scheme is indispensable. Only authentic user can access to SoR through API authentication.

\subsection{Implementation of SoR}

Previous research has been investigated related to the implementation of SoR in both analytical simulations and real practical network experiments.

One of implementation of SoR is "NEGI", a software based simulator, is created as the implementation of SoR with basic 
functionalities [26]. NEGI is a packet analyzer which analyzes data streams and compares packet streams using regular expressions. Another functionalities of the SoR is the capability to analyze packet streams and store the frequency value of accessing websites and pdf files [27]. Such information can be used to provide recommendations for web sites and pdf files for clients. In addition, hardware architecture for supporting high-speed database insertion for SoR is proposed in order to make SoR has capability to handle a gigabit-speed packet transmitted in future internet [28]. In addition, it is possible to put an intelligent capability inside SoR such as Bayesian network [29, 30] or other AI tools.

SoR has been proposed as a router-core in redirection mechanism in CDN [31]. Furthermore, this paper proposed a further discussion about router-based redirection in CDN using SoR as a core-router with modelling investigation and test bed experiment.

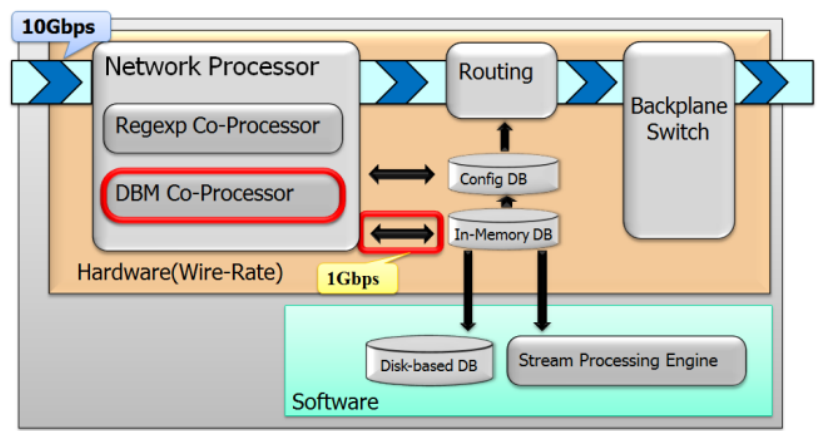

Fig 4: System architecture of Service-oriented Router

\section{REQUEST REDIRECTION}

\subsection{Router-based redirection}

We designed and modeled a router-based request redirection using SoR as core-router. Based on the functions of SoR described in section 3, a SoR model is designed where SoR has capability to redirect request form clients to the best server with minimum cost (server loads, network delay, server processing time, etc.). In this model, the following factors are considered: for a given workload (mean of service time, and network delay), an expression of redirection probability is derived to minimize the response time so that SoR redirect request to the best server. Next, the average response time including service rate is computed and network delay. Then, a systematic performance analysis is performed to estimate the optimal redirection probability $\alpha *$ predict the expected average response time of request under varying parameters: server loads, network delay, and service rate of SoRs.

Figure 5 illustrates the system model for CDN with routerbased redirection. Packet streams request come from cluster client $i$ to a particular destination surrogate server $j$ through SoR $i$, which both of its header and payload is analyzed. Since SoRs communicate between each other as well as communicate with servers, SoR are aware of the best destination to forward the packet requests. (communication mechanism between SoR-SoR and SoR-Server is not discussed in this paper). We modeled the system as a M/M/1 queuing model, where request packet-arrivals from client is a Poisson process with rate $\lambda$ and by an exponential distribution service time $\mu$.

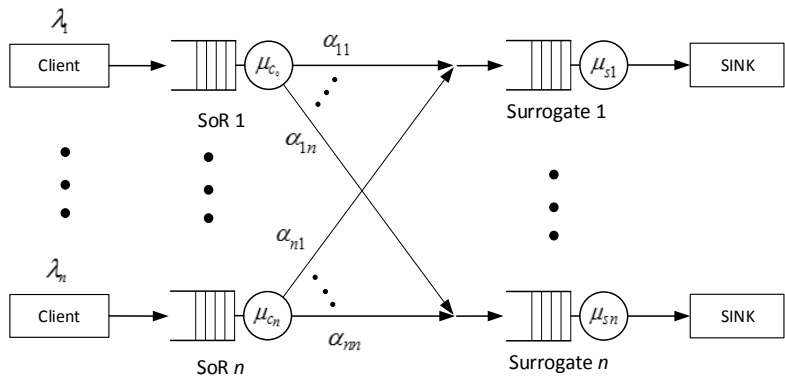

Fig 5: Model architecture of CDN with router-based request redirection

We consider a redirection algorithm in which a request is redirected to the best server with probability $\alpha_{i j}$. In this case, a probabilistic redirection is considered. We also denote $R$ as a response time experienced by packet sent from a particular client, and $E[T]$ as the expected response time for servicing a request in the system. In addition, $D$ is denoted as a one-way end-to-end network latency between nodes. For the general case of a system of $n$ SoRs, and $n$ servers, let us denote $A=$ $\left\{\alpha_{11}, \ldots, \alpha_{i j}, \ldots, \alpha_{n n}\right\}$ as a matrix of request redirection probability from SoR to surrogate servers, $R=\left\{R_{1}, \ldots, R_{n}\right\}$ as the vector of total average of system response time for a particular request, $E[T]=\left\{T_{1}, \ldots, T_{n}\right\}$ as the vector of system response time in a particular node, and $\mathrm{E}[D]=\left\{d_{12}, \ldots, d_{i j}, \ldots\right.$, $\left.d_{n m}\right\}$ as matrix of network delay from node $i$ to $j$. Furthermore, $\Gamma=\left\{\gamma_{1}, \ldots, \gamma_{n}\right\}$ is denoted as a vector of total input of request arrival rates in to the system, $L=\left\{\lambda_{1}, \ldots, \lambda_{n}\right\}$ is denoted as a vector of packet arrival rates at particular node, $X^{S O R}=$ $\left\{\mu_{1}^{S O R}, \ldots, \mu_{n}^{S O R}\right\}$ as the service rate in $\operatorname{SoR}, X^{S S}=$ $\left\{\mu_{1}^{S S}, \ldots, \mu_{n}^{S S}\right\}$ as the service rate in surrogate server.

Theorem 1. The expected response time in the system using a redirection policy $A$ is given by:

$$
R=E[T]+E[D]
$$

where

$$
E[T]=\frac{1}{\Gamma}\left(\frac{L}{X^{S o R}-L}+\frac{A L}{X^{S S}-A L}\right)
$$

and

$$
E[D]=D^{S o R}+A D^{S S}
$$

Proof: The total response time of a particular request by routerbased redirection method are composed of 3 durations: (i) response time in SoR, (ii) response time in surrogate server, and (iii) network delay from client to SoR and from SoR to surrogate server. Design architecture depicted on figure 1 considered as a queuing networks with tandem servers. Refer to Jackson Networks with M/M/1 queuing model [14, 32, 33], the average number of customer in in every terminal/nodes in the system is given by

$$
N=\sum_{i=1}^{M} \frac{\rho_{i}}{1-\rho_{i}}
$$

with $\rho=\lambda / \mu$, then 


$$
N=\sum_{i=1}^{M} \frac{\lambda_{i}}{\mu_{i}-\lambda_{i}}
$$

According to little theorem, system response time of the system is given by

$$
T=\frac{N}{\sum_{i=1}^{n} \gamma_{i}}=\frac{1}{\sum_{i=1}^{n} \gamma_{i}} \sum_{i=1}^{n} \frac{\lambda_{i}}{\mu_{i}-\lambda_{i}}
$$

Refer to figure 1, the system is consist of SoRs and Surrogate Servers, (6) can be rewritten as:

$$
T=\frac{1}{\sum_{i=1}^{n} \gamma_{i}} \sum_{i=1}^{n}\left(\frac{\lambda_{i}^{S o R}}{\mu_{i}^{S o R}-\lambda_{i}^{S o R}}+\frac{\lambda_{i}^{S S}}{\mu_{i}^{S S}-\lambda_{i}^{S S}}\right)
$$

Let client $i$ sent a packet request with arrival rate $\lambda_{i}$. According to figure 1 , arrival rate in SoR is equal with arrival rate generated from client $i, \lambda_{i}^{(S O R)}=\lambda_{i}$. Let $\propto_{i j}$ is a redirection probability from SoR $i$ to surrogate $j$. Since every packet is analyzed in SoR, and then it takes decision to redirect to the best surrogate server, then arrival rate in surrogate server is depend on the probability $\propto_{i j}$ such that $\lambda_{i}^{(S S)}=\propto_{i j} \lambda_{i}$. Equation (7) can be rewritten as:

$$
\begin{array}{r}
T=\frac{1}{\sum_{i=1}^{n} \gamma_{i}} \sum_{i=1}^{n}\left(\frac{\lambda_{i}}{\mu_{i}^{S o R}-\lambda_{i}}+\sum_{j=1}^{n} \frac{\alpha_{i j} \lambda_{i}}{\mu_{i}^{S S}-\alpha_{i j} \lambda_{i}}\right) \\
T_{i}=\frac{1}{\gamma_{i}}\left(\frac{\lambda_{i}}{\mu_{i}^{S o R}-\lambda_{i}}+\sum_{j=1}^{n} \frac{\alpha_{i j} \lambda_{i}}{\mu_{i}^{S S}-\alpha_{i j} \lambda_{i}}\right)
\end{array}
$$

Next, consider a one way latency when transmitting packet request from client $i$ to SoR $i$ by $d_{i}^{S o R}$ and from SoR $i$ to surrogate server $j$ by $d_{i j}^{S S}$ such that the latency from client to surrogate can be written as

$$
\begin{gathered}
D=\sum_{i=1}^{n}\left(d_{i}^{S o R}+\sum_{j=1}^{n} \alpha_{i j} d_{i j}^{S S}\right) \\
D_{i}=d_{i}^{S o R}+\sum_{j=1}^{n} \alpha_{i j} d_{i j}^{S S}
\end{gathered}
$$

From (8) and (9), the total response time given by

$$
R=\sum_{i=1}^{n}\left(T_{i}+D_{i}\right)
$$

from equation (9), let $E[D]$ as a vector of $D_{i}$ for $i, j=1, \ldots, n$ yields equation (3). In addition, equation (8), let $E[T]$ as a vector of $T_{i}$ for $i=1, \ldots, n$ yields equation (2), and finally from equation (10), let $E[R]$ as a vector of $R_{i}$ for $i=1, \ldots, n$ yields equation (1).

From equation (1), the optimal redirection probability is computed so that it minimize the response time over all requests. In particular, let $A^{*}=\left\{\alpha_{11}, \ldots, \alpha_{i j}, \ldots, \alpha_{n n}\right\}$ denote the matrix of optimal request redirection probability. The optimal redirection probability $A$ are given by differentiating the equation (1) such that

$$
\frac{\partial}{\partial \alpha} R=\frac{\partial}{\partial \alpha}(E[T]+E[D])=0
$$

decompose equation (11) and reduce variables without $\propto$, the equations can be re-written as

$$
\frac{\partial}{\partial \alpha}\left(\frac{A L}{\Gamma\left(X^{S S}-A L\right)}+A D\right)=0
$$

To solve equation (12), for all $\propto_{i j}$, a set of simplifying assumptions is created to reduce the number of unknowns variables. First, it is clear that $\sum_{i} \propto_{i j}=1$. Second, assumed that, all surrogate server have equal service rate. Third, if $\lambda_{i} \leq \lambda_{j} \Rightarrow \propto_{i j^{*}}=0$. When considering 2 surrogate server with different $\lambda$. Fourth, if $d_{i} \leq d_{j} \Rightarrow \propto_{i j} *=0$, subject to redirect request to the shortest distance. Under steady state conditions, no requests will be dispatched from SoRs to surrogate server with higher cost, where cost is defined as a joint delay of surrogate server service rate and network delay.

The optimal redirection probability $A^{*}$ can be used to predict the average response time for system with router-based redirection. The equation is given by

$$
R^{*}=\frac{1}{\Gamma}\left(\frac{L}{X^{S o R}-L}+\frac{A^{*} L}{X^{S S}-A^{*} L}\right)+D^{S o R}+A^{*} D^{S S}
$$

\subsection{DNS-based redirection}

To compare the performance result of the system, a CDN with DNS-based request redirection is considered. Figure 6 illustrates the system model for CDN with DNS-based redirection. Requests come from client $i$, (or cluster client $i$ ) to particular destination surrogate server $j$, through router $i$. Each of packet sent from client, will be sent to local DNS to find the destination with the best location. The system modeled as a $\mathrm{M} / \mathrm{M} / 1$ queuing model, where request arrivals from client is a Poisson process with rate $\lambda$ and by an exponential distribution service time $\mu$.

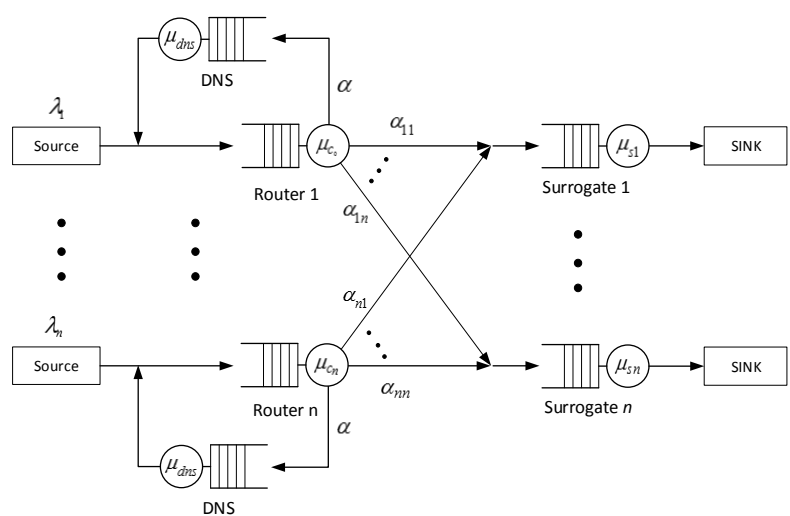

Fig 6: Model architecture of CDN with router-based request redirection 
We consider a redirection algorithm in which a request is redirected to a nearest surrogate with probability $\alpha_{i j}$. Similar with SoR system, $E[T]$ is also denoted as the expected response time for servicing a request in the system. In addition, $D$ is denoted as the delay. For the general case of a system of CDN with $n$ surrogates, let us denote $A=\left\{\alpha_{11}, \ldots, \alpha_{i j}, \ldots, \alpha_{n n}\right\}$ as a matrix of request redirection probability from SoR to surrogate server, $E[T]=\left\{T_{1}, \ldots, T_{n}\right\}$ as the vector of system mean response time, and $D=\left\{d_{12}, \ldots, d_{i j}, \ldots, d_{n m}\right\}$ as a matrix of network delay from node $i$ to node $j, L=\left\{\lambda_{1}, \ldots, \lambda_{n}\right\}$ as a vector of request arrival rates at client, $X^{R}=\left\{\mu_{1}^{R}, \ldots, \mu_{n}^{R}\right\}$ as the service rate in router, $X^{D N S}=\left\{\mu^{D N S}, \ldots, \mu^{D N S}\right\}$ as the service rate in DNS server, $X^{S S}=\left\{\mu_{1}^{S S}, \ldots, \mu_{n}^{S S}\right\}$ as the service rate in surrogate server.

Theorem 2. The expected response time for the system of DNS-based redirection policy in CDN, depicted in figure 6 , using a redirection policy $A$ is given by:

$$
R=E[T]+E[D]
$$

where

$$
E[T]=\frac{1}{\Gamma}\left(\frac{L}{X^{R}-L}+\frac{A_{1} L}{X^{D N S}-A_{1} L}+\frac{A_{2} L}{X^{S S}-A_{2} L}\right)
$$

and

$$
E[D]=D^{S o R}+A_{1} D^{D N S}+A_{2} D^{S S}
$$

Proof: Different with theorem 1, the total response time is composed of 4 durations: (i) response time in router, (ii) response time DNS, (iii) response time in surrogate server (SS), and (iii) network delay from client to router, router to DNS and from router to surrogate server. Design architecture depicted on figure 6 , considered as a queuing networks with tandem servers with $\mathrm{M} / \mathrm{M} / 1$ queuing model. The system is consist of routers, DNS servers, and surrogate servers. Similarly with proof explanation in theorem 1, and refer to Jackson network with $\mathrm{M} / \mathrm{M} / 1$ queuing networks, system response time is given by:

$$
\begin{aligned}
T= & \frac{1}{\sum_{i=1}^{n} \gamma_{i}} \sum_{i=1}^{n}\left(\frac{\lambda_{i}^{(S o R)}}{\mu_{i}^{(S o R)}-\lambda_{i}^{(S o R)}}+\frac{\lambda_{i}^{(D N S)}}{\mu^{(D N S)}-\lambda_{i}^{(D N S)}}\right. \\
& \left.+\frac{\lambda_{i}^{(S S)}}{\mu_{i}^{(S S)}-\lambda_{i}^{(S S)}}\right)
\end{aligned}
$$

Let client $i$ sent a packet request with arrival rate $\lambda_{i}$. According to figure 6 , arrival rate in router is equal with arrival rate generated from client $i, \lambda_{i}^{R}=\lambda_{i}$. Let $\propto_{i}$ is a redirection probability from router $i$ to DNS server (root, low-level DNS, or high-level DNS), $\propto_{i j}$ is a redirection probability from router $i$ to surrogate $j$. DNS server gives the appropriate destination of address so that the client's request is redirected to the best surrogate server. According to this condition, arrival rate in surrogate server is depend on the probability $\propto_{i j}$ such that $\lambda_{i}^{(S S)}=\propto_{i j} \lambda_{i}$. Equation (17) can be rewritten as:

$$
\begin{aligned}
T= & \frac{1}{\sum_{i=1}^{n} \gamma_{i}} \sum_{i=1}^{n}\left(\frac{\lambda_{i}^{S o R}}{\mu_{i}^{S o R}-\lambda_{i}^{S o R}}+\right. \\
& \left.\frac{\alpha^{D N S} \lambda_{i}}{\mu^{D N S}-\left(\alpha^{D N S} \lambda_{i}\right)}+\sum_{j=1}^{n} \frac{\alpha_{i j}^{S S} \lambda_{i}}{\mu_{i}^{S S}-\alpha_{i j}^{S S} \lambda_{i}}\right) \\
T_{1}= & \frac{1}{\gamma_{i}}\left(\frac{\lambda_{i}^{S o R}}{\mu_{i}^{S o R}-\lambda_{i}^{S o R}}+\right. \\
& \left.\frac{\alpha^{D N S} \lambda_{i}}{\mu^{D N S}-\left(\alpha^{D N S} \lambda_{i}\right)}+\sum_{j=1}^{n} \frac{\alpha_{i j}^{S S} \lambda_{i}}{\mu_{i}^{S S}-\alpha_{i j}^{S S} \lambda_{i}}\right)
\end{aligned}
$$

Next, consider a one way latency when transmitting packet request from client $i$ to router $i$ by $d_{i}^{R}$, from router $i$ to DNS server by $d^{D N S}$, and from router $i$ to surrogate server $j$ by $d_{i j}^{S S}$ such that the latency from client to surrogate can be written as

$$
\begin{gathered}
D=\sum_{i=1}^{n}\left(d_{i}^{R}+\alpha_{i} d^{D N S}+\sum_{j=1}^{n}\left(\alpha_{i j} d_{i j}^{S S}\right)\right) \\
D_{i}=\left(d_{i}^{R}+\alpha_{i} d^{D N S}+\sum_{j=1}^{n}\left(\alpha_{i j} d_{i j}^{S S}\right)\right)
\end{gathered}
$$

From (18) and (19), the total response time, can be written as

$$
R=\sum_{i=1}^{n}\left(T_{i}+D_{i}\right)
$$

from equation (19), let $E[D]$ as a vector of $D_{i j}$ for $i, j=1, \ldots, n$ yields equation (16). In addition, equation (18), let $E[T]$ as a vector of $T_{i}$ for $i=1, \ldots, n$ yields equation (15), and finally from equation (20), let $E[R]$ as a vector of $R_{i}$ for $i=1, \ldots, n$ yields equation (14).

From equation (14), the optimal redirection probability that minimize the response time over all requests can be calculated. In particular, let $A^{*}=\left\{\alpha_{11}, \ldots, \alpha_{i j}, \ldots, \alpha_{n n}\right\}$ denote the matrix of optimal request redirection probability. The optimal redirection probability $A$ are given by differentiating the equation (1) such that

$$
\frac{\partial}{\partial \alpha^{S S}} R=\frac{\partial}{\partial \alpha^{S S}}(E[T]+E[D])=0
$$

decompose equation (21) and reduce variables without $\propto^{S S}$, the equations can be re-written as

$$
\frac{\partial}{\partial \alpha^{S S}}\left(\frac{A_{2} L}{\Gamma\left(\bar{X}^{(S S)}-A_{2} L\right)}+A_{2} D\right)=0
$$

to solve equation (22), for all $\propto_{i j}$, a set of simplifying assumptions is created to reduce the number of unknowns variables. First, it is clear that $\sum_{i} \propto_{i j}=1$. Second, all surrogate servers are assumed have equal service rate. Third, if $\lambda_{i} \leq \lambda_{j} \Rightarrow \propto_{i j} *=0$. When considering 2 surrogate server with different $\lambda$. Fourth, if $d_{i} \leq d_{j} \Rightarrow \propto_{i j} *=0$, subject to redirect 
request to the shortest distance. Under steady state conditions, no requests will be dispatched from router to surrogate server with higher cost, where cost is defined as a joint delay of surrogate server service rate and network delay.

The optimal redirection probability $A^{*}$ can be used to predict the average response time for system with DNS-based redirection. The equation is given by

$$
\begin{aligned}
R^{*}= & \frac{1}{\Gamma}\left(\frac{L}{X^{R}-L}+\frac{A_{1}^{*} L}{X^{D N S}-A_{1}^{*} L}+\frac{A_{2}^{*} L}{X^{S S}-A_{2}^{*} L}\right) \\
& +D^{R}+A_{1}^{*} D^{D N S}+A_{2}^{*} D^{S S}
\end{aligned}
$$

Equation (23) follows from equation (14) and by using the optimal redirection probability from equation (22).

\subsection{Numerical Analysis}

In this section, the router-side redirection method is provided with its performance result on reduction of the total response time. Then, the effect of the key performance factor that affect the total delay is studied and analyzed. Consider a system consist of 1 client, 1 SoR, and 2 surrogate server having same service rate. Furthermore, let $d_{1}$ is a network delay between client and SoR, and $d_{i j}^{S S}$ is network delay between SoR and surrogate server. In addition, given $\lambda_{1}^{S S}>\lambda_{2}^{S S}$ and $d_{11}^{S S}>d_{12}^{S S}$ which satisfies $\alpha_{11}=0$ and denote $\alpha^{*}=\alpha_{12}^{*}$. The system response time is computed based on theorem 1 :

$$
\begin{aligned}
R_{1}= & \frac{1}{\gamma}\left(\frac{\lambda_{1}}{\mu_{1}^{S o R}-\lambda_{1}}+\frac{\alpha_{11} \lambda_{1}}{\mu_{1}^{S S}-\alpha_{11} \lambda_{1}}+\frac{\alpha_{12} \lambda_{1}}{\mu_{2}^{S S}-\alpha_{12} \lambda_{1}}\right) \\
& +d^{S o R}+\alpha_{11} d_{11}^{S S}+\alpha_{12} d_{12}^{S S}
\end{aligned}
$$

since $\alpha_{11}+\alpha_{12}=1$, equation (24) can be rewritten as

$$
\begin{aligned}
& R_{1}=\frac{1}{\gamma}\left(\frac{\lambda_{1}}{\mu_{1}^{S o R}-\lambda_{1}}+\frac{\alpha_{11} \lambda_{1}}{\mu_{1}^{S S}-\alpha_{11} \lambda_{1}}+\right. \\
& \left.\frac{\left(1-\alpha_{11}\right) \lambda_{1}}{\mu_{2}^{S S}-\left(1-\alpha_{11}\right) \lambda_{1}}\right)+d^{S o R}+\alpha_{11} d_{11}^{S S}+\left(1-\alpha_{11}\right) d_{12}^{S S}
\end{aligned}
$$

Equation (25) are solved according to equation (12) to obtain the optimal redirection probability. We refer to the term $\alpha^{*}$ as the optimal redirection probability from SoR to the default surrogate server, and $1-\alpha^{*}$ is the redirection probability to other surrogate server. Then, according to the equation (13), the response time of the router-based redirection system is given by

$$
\begin{aligned}
& R_{1}^{*}=\frac{1}{\gamma}\left(\frac{\lambda_{1}}{\mu_{1}^{S o R}-\lambda_{1}}+\frac{\alpha_{11}^{*} \lambda_{1}}{\mu_{1}^{S S}-\alpha_{11}^{*} \lambda_{1}}+\right. \\
& \left.\frac{\left(1-\alpha_{11}^{*}\right) \lambda_{1}}{\mu_{2}^{S S}-\left(1-\alpha_{11}^{*}\right) \lambda_{1}}\right)+d^{S o R}+\alpha_{11}^{*} d_{11}^{S S}+\left(1-\alpha_{11}^{*}\right) d_{12}^{S S}
\end{aligned}
$$

The same way is applied to calculate the response time of the DNS-based redirection system. Consider a system refer to figure 2 consist of 2 clients, 2 routers, 1 DNS server, and 2 surrogate servers. We assume a network delay between of client and router (d1), router and DNS server, router and first surrogate server (d3), router and second surrogate server (d4).
Similar with router-based redirection system, given $\lambda_{1}^{S S}>\lambda_{2}^{S S}$ and $d_{11}^{S S}>d_{12}^{S S}$ which satisfies $\alpha_{11}=0$ and denote $\alpha^{*}=\alpha_{12}^{*}$. The response time is computed based on theorem 2

$$
\begin{aligned}
& R_{1}=\frac{1}{\gamma_{1}}\left(\frac{\lambda_{1}}{\mu_{1}^{R}-\lambda_{1}}+\frac{\alpha_{1}^{D N S} 3 \lambda_{1}}{\mu_{1}^{D N S}-\alpha_{1}^{D N S} 3 \lambda_{1}}+\frac{\alpha_{11} \lambda_{1}}{\mu_{1}^{S S}-\alpha_{11} \lambda_{1}}\right. \\
& \left.+\frac{\alpha_{12} \lambda_{1}}{\mu_{2}^{S S}-\alpha_{12} \lambda_{1}}\right)+d^{R}+\alpha^{D N S} d^{D N S}+\alpha_{11} d_{11}^{S S}+\alpha_{12} d_{12}^{S S}
\end{aligned}
$$

Equation (27) are solved according to equation (22) to obtain the optimal redirection probability. We refer to the term $\propto^{*}$ as the optimal redirection probability from SoR to the default surrogate server, and $1-\propto^{*}$ is the redirection probability to other surrogate server. Then, according to the equation (23), the response time of the router-based redirection system is given by:

$$
\begin{aligned}
& R_{1}^{*}=\frac{1}{\gamma_{1}}\left(\frac{\lambda_{1}}{\mu_{1}^{R}-\lambda_{1}}+\frac{\alpha_{1}^{D N S} 3 \lambda_{1}}{\mu_{1}^{D N S}-\alpha_{1}^{D N S} 3 \lambda_{1}}+\frac{\alpha_{11}^{*} \lambda_{1}}{\mu_{1}^{S S}-\alpha_{11}^{*} \lambda_{1}}\right. \\
& \left.+\frac{\left(1-\alpha_{11}^{*}\right) \lambda_{1}}{\mu_{2}^{S S}-\left(1-\alpha_{11}^{*}\right) \lambda_{1}}\right)+d^{R}+\alpha_{1}^{D N S}\left(d_{1}^{D N S_{1}}+d_{1}^{D N S_{2}}\right) \\
& +\alpha_{11}^{*} d_{11}^{S S}+\left(1-\alpha_{11}^{*}\right) d_{12}^{S S}
\end{aligned}
$$

\section{ANALYTICAL SIMULATION EXPERIMENT}

\subsection{Redirection probability and Response time}

Based on the theorems and equations given in section VI, an experiment is conducted to simulate the aforementioned theorems. We use default values as follow: the average of network delay between client, $d_{1}$, and router or SoR (measured in one cluster of PlanetLab network testing environment), is $0.5 \mathrm{~ms}$. Average delay from SoR/router to DNS server, $d_{2}$, is $30 \mathrm{~ms}$, taken by using DNS take records [9]. Network delay between SoR/router to surrogate server, $d_{3}$, is $36 \mathrm{~ms}$ [5]. Packet size is 1500 bytes. Service rates for routers, SoRs, DNS servers, and Surrogate servers are listed in table 1. We set router's service rate is $10 \mathrm{Mbps}$. Since SoR has additional functions compared to a regular router, SoR's processing time might be longer than regular router. Therefore, SoR's service rate is set as $50 \%$ of regular router, or two times slower. DNS server' service rate is set as equal as a regular router.

Table 1. Network node's service rate

\begin{tabular}{|l|r|r|}
\hline \multirow{2}{*}{\multicolumn{1}{|c|}{ Nodes }} & \multicolumn{2}{c|}{ Service rate $(\boldsymbol{\mu})$} \\
\cline { 2 - 3 } & \multicolumn{1}{c|}{ bps } & packet per second \\
\hline Router & $10 \mathrm{Mbps}$ & 830 packet per second \\
\hline SoR & $5 \mathrm{Mbps}$ & 415 packet per second \\
\hline DNS server & $10 \mathrm{Mbps}$ & 830 packet per second \\
\hline Surrogate Server & $1 \mathrm{Mbps}$ & 83 packet per second \\
\hline
\end{tabular}

In this experiments, the evidence that router-based redirection is able to decrease the user-perceived response time and total delay is provided. The response time of router-based redirection using equation (13) is calculated and compared to 
the response time of DNS-based redirection, using equation (23). Two surrogate servers are given that have the same contents and loads. Surrogate server\#1 placed with fix network delay (36 ms) from SoR or Router. Surrogate server\#2 placed dynamically from 0 to $250 \mathrm{~ms}$ delay. Figure (7) shows response time as a function of the network delay of surrogate server for different system loads $\rho$.

For the given surrogate server loads; $\rho=0.50, \rho=0.75$, and $\rho=0.90$, improvements of routing redirections are achieved by router-based redirection compared with DNS-based redirection. The differences of response time between routerbased redirection and DNS-based redirection is 11 to $14 \mathrm{~ms}$.

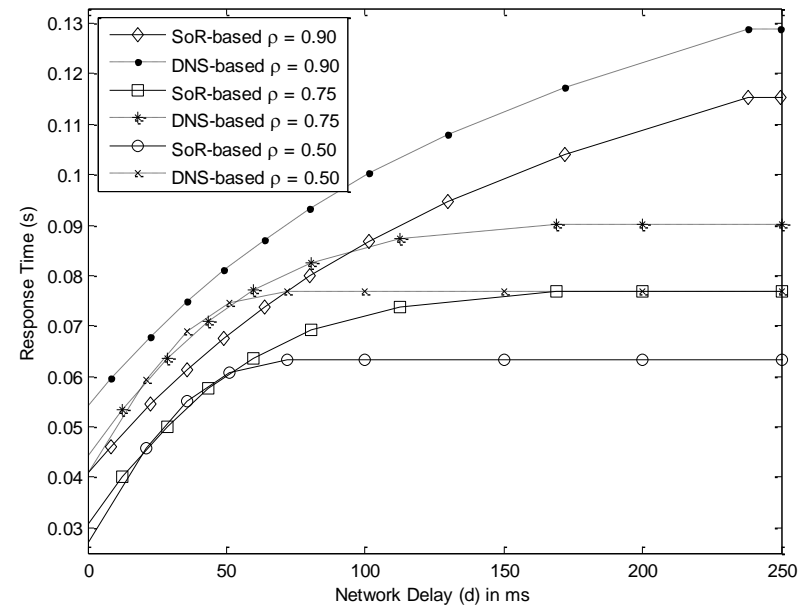

Fig 7: Total response time based on the delay between router or SoR to server

For loads $\rho_{S O R}=0.50$ and $\rho_{D N S}=0.50$, when network delay of surrogate server\#2 less than $75 \mathrm{~ms}\left(d_{3}^{S S 2} \leq 75 \mathrm{~ms}\right)$, most of request is redirected to nearest surrogate server. Based on the redirection probability given calculated by equation (11) and (21), that given in figure 8, the server's destination could be Surrogate server\#1 or Surrogate Server\#2.

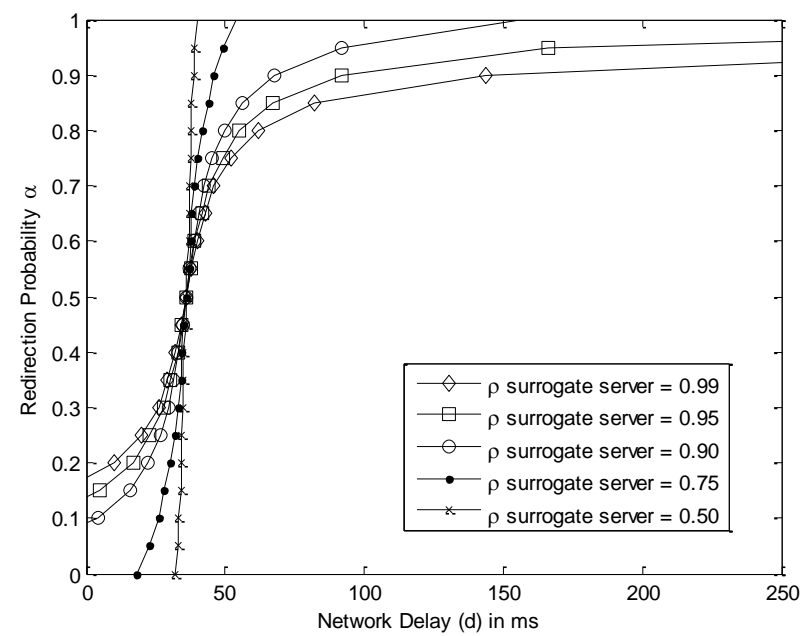

Fig 8: Redirection probability based on the delay between router (or SoR) to server

For network delay of surrogate server\#2 more than $75 \mathrm{~ms}$, the redirection cost exceeds the processing time such that all requests are served by surrogate server\#1, as the nearest destination. Based on the experiment result in figure 7 , the cause of higher response time of DNS-based redirection policy is generally because of network delay between router and DNS-server.

As explained in section 3, in router-based redirection method is able to redirect the client's requests from the router (SoR) itself, while in DNS-based redirection method, requests have to wait until DNS-server resolve the appropriate destination server. That phenomenon literally slow down the DNS-based redirection method compared to the router-based redirection method.

\section{DISCUSSION}

We validate the analytical model of Section 3 and system performance result in section 4 for both response time and redirection probabilities. In particular, the router-based and DNS-based redirection is compared for total response times with two servers. For the model, Equations (1) and (13) as well as Equation (14) and (23) are used with $\mu_{R}=10 \mathrm{Mbps}$ and $\mu_{S o R}=5 \mathrm{Mbps}$. Figure 7 compares the average of response time of the model regarding to the implementation of CDN with two servers. The figure indicates that the model matches the measured response time for $\rho_{S S 1}, \rho_{S S 2}$ of each servers. With this server load, our $\mathrm{M} / \mathrm{M} / 1$ model give the analytical measurement the theory of Jackson networks in queuing networks [14], that described the system in steady state situation. Next, the model with the two implemented redirection policies are compared: (1) router-based request redirection, and (2) DNS-based request redirection. In the router-based redirection method, the service-rate of SoR is set to be $50 \%$ of regular router service rate in DNS-based redirection. The result indicate that for any given server loads, the differences of response time of router-based and DNSbased redirection are always same, that is from $11 \mathrm{~ms}$ to $14 \mathrm{~ms}$. With this result, in steady state situation the weakness of DNSbased redirection is because of the network delay. The packet stream request should travel from client to the DNS-server and travel back to the client until finally it sent to the proper destination. In router-based redirection, the packet stream sent directly to the proper destination, and it reduce the response time.

Figures 8 is the redirection probability as a function of the server's network delay. Based on equation (11) and (21), the values of redirection probability calculated from the routerbased and DNS-based redirection are equal. The values are equal because of the request redirection method is bases upon optimal values predicted by the model on the range or delay between router/SoR to the destination server. Since the redirection value is the function of the server's network delay, any loads changes within all network nodes will not affect the value of redirection probability.

\section{CONCLUSION}

CDNs deal with a communication process where network latency and server capacity are decisive parameters in the response time perceived by the user, as well as the system's client redirection capability to match each client with a suitable nearest servers. Performance studies have been made in our research both in empirical and analytical approaches. We introduced a Service-oriented Router (SoR) as an alternative of core router to improve $\mathrm{CDN}$ architecture with embedding more functional routers. We modeled an $\mathrm{M} / \mathrm{M} / 1$ queuing system in steady state environment. The experiment result shows that with $50 \%$ times service rate of SoR compare to regular router, and optimal redirection probability between two servers, the reduction of response time of router-based redirection using 
SoR is achieved by 11 to $14 \mathrm{~ms}$ or $23.3 \%$ compared to DNSbased redirection.

A further research is intended to do with different probabilityweight assignment policies for router-based redirection. Dynamically changing the probability are more desirable following with artificial intelligent such as Bayesian network or neural network. Complementary models of server migration mechanisms, content placement, and load balancing mechanisms are to be introduced in future work.

\section{ACKNOWLEDGMENTS}

This work was partially supported by Funds for integrated promotion of social system reform and research and development, MEXT, Japan, by Low Carbon Technology Research and Development Program for "Practical Study on Energy Management to Reduce $\mathrm{CO} 2$ emissions from University Campuses" from Ministry of the Environment, Japan and by MEXT/JSPS KAKENHI Grant (B) Number 24360230 and 25280033. In addition, this work is supported by Directorate General of Higher Education (DGHE/DIKTI), Ministry of National Education of Indonesia (E24/2011-2014).

\section{REFERENCES}

[1] George Pallis, Athena Vakali., "Insight and Perspectives for Content Delivery Networks," Journal Communication of the ACM, vol. 49, pp. 101-106, 2006.

[2] "Akamai," [Online]. Available: http://www.akamai.com. [Accessed June 2013].

[3] "Limelight Networks," [Online]. Available: http://www.limelight.com/. [Accessed July 2013].

[4] "Mirror Image," [Online]. Available: http://www.mirror-image.com/. [Accessed July 2013].

[5] S. Ranjan, "Request Redirection for Dynamic Content," in Content Delivery Network, vol. XVI, Springer, 2008, pp. 155-182.

[6] Magnus Karlsson, Mallik Mahalingam, "Do we need replica placement algorithms in content delivary networks," in 7th International Workshop on Web Content Caching and Distribution (WCW), 2002.

[7] Limin Wang, Vivek Pai, Larry Peterson, "The effectiveness of request redirection on CDN robustness," in 5th symposium on Operating systems design and implementation (ACM SIGOPS), 2002.

[8] Dilley, J., Maggs, B., Parikh, J., Prokop, H., Sitaraman, R., Welhl, B. , "Globally distributed content delivery," in IEEE Internet Computing, 2002.

[9] Shaikh, A.; Tewari, R.; Agrawal, M.; "On the effectiveness of dns-based server selection," in Twentieth Annual Joint Conference of the IEEE Computer and Communications Societies (IEEE INFOCOM), 2001.

[10] Ranjan, S., Karrer, R., Knightly, E., "Wide area redirection of dynamic content in internet data centers," in IEEE INFOCOM, Hongkong, 2002.

[11] K. Inoue, D. Akashi, M. koibuchi, H. Kawashima, H. Nishi, Semantic router using data stream to enrich services, Seoul: 3rd International Conference on Future Internet Technologies (CFI08), 2008.
[12] "Information-based Open Innovation Platform (IOIP)," [Online]. Available: http://openinter.net/. [Accessed July 2013].

[13] "New Generation Network enabled by Service-oriented Router," [Online]. Available:

[14] http://sor.west.sd.keio.ac.jp/english/index.html. [Accessed July 2013].

[15] G. B. et.all., Queuing Networks and Markov Chains, Modeling and Performance Evaluation with Computer Science Applications, vol. 2, Hoboken: John Willey \& Sons Inc., 2006.

[16] Shaikh, A.; Tewari, R.; Agrawal, M.; "On the effectiveness od DNS-based server selection," in INFOCOM, 2001.

[17] "Science Information NETwork," [Online]. Available: http://www.sinet.ad.jp/. [Accessed July 2013].

[18] Z. Mao, C. Cranor, F. Douglis, M. Rabinovich, "A precise and efficient evaluation of the proximity of web clients and their local DNS servers," in USENIX'02, Monterey CA (USA), June, 2002.

[19] J. Kangasharju, K.W. Ross, J.W. Roberts, "Performance evaluation of redirection schemes in content distribution networks," in 5th International Workshop on Web Caching and Content Distribution, Lisbon (Portugal), June, 2000.

[20] B. Krishnamurthy, C. Wills, Y. Zhang, "On the use and performance of Content Delivery Networks," in ACM SIGCOMM Internet Measurements Workshop, San Diego (USA), August, 2001.

[21] R.P. Doyle, J.S. Chase, S. Gadde, A.M. Vahdat, "The trickle-down effect: web caching and server request distribution," in Computer Communication, 2002.

[22] P.S.M. Sayal, P. Vingralek, "Selection algorithms for replicated web servers," in ACM SIGMETRICS Internet Server Perfsormance Workshop, Madison (USA), June, 1998.

[23] C. Cameron, S. Low, D. Wei, "High-density model for server allocation and placement," in ACM SIGMETRICS'02, Marina del, Rey CA (USA), June, 2002.

[24] D. Agrawal, J. Giles, D. Verma, "On the performance of content distribution networks," in International Symposium on Performance Evaluation of Computer and Telecommunication Systems, Orlando (USA), July, 2001.

[25] M. Masa, E. Parravicini, "Impact of request routing algorithms on the delivery performance of content delivery networks," in 22nd IEEE International Performance Computing and Communications Conference, Phoenix (USA), April, 2003.

[26] S. Calo, D. Verma, D. Agrawal, J. Giles, "On the Effectiveness of Content Distribution Networks," in International Symposium on Performance Evaluation of Computer and Telecommunication Systems, San Diego (USA), July, 2002.

[27] Shinichi Ishida,Singo Harashima, Michihiro Koibuchi, Hideyuki Kawashima, Hiroaki Nishi, "A Software Implementation and Evaluation for Searching and 
Extracting Information of Application Layer from Network Traffic [in Japanese]," Computer Software Special Issue, vol. 29, no. 4, pp. 59-73, November 2012.

[28] Kazuki Masuda, Shinichi Ishida, Hiroaki Nishi., "Crosssite recommendation application based on the viewing time and contents of webpages captured by a Network Router," in ICOMP, Las Vegas, 2013.

[29] Tomoaki Makino, Koichi Inoue, Michihiro Koibuchi, Hideyuki Kawashima, Hiroaki Nishi, "Hardware Architecture for Supporting High-speed Database Insertion on Service-oriented Router for Future Internet," in Proceedings of the International Conference on Parallel and Distributed Processing Techniques and Applications (PDPTA), Las vegas, Nevada, USA., 2010.

[30] E. Harahap, W. Sakamoto, H. Nishi, "Failure prediction method for network management system by using bayesian network with shared database," in The 8th Asia
Pasific Symposium on Information and Telecommunication Technologies, APSITT, 2010.

[31] Erwin Harahap, Janaka Wijekoon, Hiroaki Nishi, "Distributed algorithm for efficient use of replica servers in content delivery network," in APSITT 9th, AsiaFasific Symposium on Information and Telecommunication Technology, Santiago, Chile, 2012.

[32] Janaka Wijekoon, Erwin Harahap, Hiroaki Nishi, "SoR based Request Routing for CDN," in Application of Information and Communication Technology, Tbilisi, Georgia, 2012.

[33] Ng, Chee-Hock and Boon-Hee, Soong, Queueing Modelling Fundamentals: With Applications in Communication Networks, 2 ed., England: John Wiley \& Sons Ltd Publishing Ltd., 2008.

[34] L. Kleinrock, Queuing Systems, vol. 1, Canada: John Wiley \& Sons, Inc., 1975. 